\title{
Fault Diagnosis of Rotating Machinery Based on Multisensor Information Fusion Using SVM and Time-Domain Features
}

\author{
Ling-li Jiang, ${ }^{1,2}$ Hua-kui Yin, ${ }^{1}$ Xue-jun $\mathrm{Li}^{2}{ }^{2}$ and Si-wen Tang ${ }^{1}$ \\ ${ }^{1}$ Engineering Research Center of Advanced Mining Equipment, Ministry of Education, \\ Hunan University of Science and Technology, Xiangtan 411201, China \\ ${ }^{2}$ Hunan Provincial Key Laboratory of Health Maintenance for Mechanical Equipment, \\ Hunan University of Science and Technology, Xiangtan 411201, China
}

Correspondence should be addressed to Ling-li Jiang; linlyjiang@163.com

Received 5 August 2012; Accepted 27 November 2012; Published 8 April 2014

Academic Editor: Valder Steffen

Copyright (C) 2014 Ling-li Jiang et al. This is an open access article distributed under the Creative Commons Attribution License, which permits unrestricted use, distribution, and reproduction in any medium, provided the original work is properly cited.

\begin{abstract}
Multisensor information fusion, when applied to fault diagnosis, the time-space scope, and the quantity of information are expanded compared to what could be acquired by a single sensor, so the diagnostic object can be described more comprehensively. This paper presents a methodology of fault diagnosis in rotating machinery using multisensor information fusion that all the features are calculated using vibration data in time domain to constitute fusional vector and the support vector machine (SVM) is used for classification. The effectiveness of the presented methodology is tested by three case studies: diagnostic of faulty gear, rolling bearing, and identification of rotor crack. For each case study, the sensibilities of the features are analyzed. The results indicate that the peak factor is the most sensitive feature in the twelve time-domain features for identifying gear defect, and the mean, amplitude square, root mean square, root amplitude, and standard deviation are all sensitive for identifying gear, rolling bearing, and rotor crack defect comparatively.
\end{abstract}

\section{Introduction}

Typical rotating machinery systems such as water turbine, steam turbine, wind turbine, and rotary kiln are critical core equipment support of the important industries of the national economy $[1,2]$. The safety, reliability, efficiency, and performance of rotating machinery are major concerns in industry, so, the task of condition monitoring and fault diagnosis of rotating machinery is significant [3]. The common mechanical defects of rotating machinery are divided into three categories: (1) rotor body defects, such as unbalance, misalignment, rubbing, and rotor crack; (2) rotor supportbearing defects, such as inner race, outer race or ball defect of rolling bearing, and oil whirl or oil whip of sliding bearing; (3) transmission gear defects, such as chipped tooth defect or missing tooth defect. In-process monitoring and diagnostics of rotating machinery require reasoning about defect and process states from sensor readings. Often the relationship between the sensor readings and the process states is complex and nondeterministic. For a complex system, a single sensor is incapable of collecting enough data for accurate condition monitoring and fault diagnosis. Multiple sensors are needed in order to do a better job. When multiple sensors are used, data collected from different sensors may contain different partial information about the same machine condition. The diagnostic object can be described more comprehensively [46]. Compared with single sensor, the time-space scope and the quantity of information are expanded. The diagnostic accuracy and reliability can be improved. Multisensor information fusion can be categorized into three levels [7, 8]: datalevel fusion, feature-level fusion, and decision-level fusion.

At data-level fusion, all sensor data from a measured object are combined directly and features are then calculated from the fused data. Fusion of data at this level contains most information and can deliver good results. However, the sensors used in this level must be commensurate. That means 
the measurement has to be the same or has similar physical quantities or phenomena. During the most popular datalevel fusion methodology, such as weighted fusion [9], the weighted value of multisensor signals is difficult to determine. As a consequence, data-level applications are limited in real environment. At feature-level fusion, the features are calculated from each sensor according to the type of raw data. Then, these noncommensurate sensors features are combined at the feature level. All features are combined in turn into a bigger single feature set, which are then used in a special classification model such as artificial neural network (ANN), support vector machine (SVM), and cluster algorithm for decisions [10]. The feature-level fusion is a compromise form of data-level fusion and decision-level fusion. Its data alignment requirements are not strict as the data-level fusion that heterogeneous sensors are allowed, and its information loss is less serious than the decision-level fusion but still achieved a better information compression. As a consequence, feature-level applications are flexible and popular. At decision-level fusion, the processes of features calculation and pattern recognition are applied in sequence for single-source data obtained from each sensor. The decision vectors are then fused using decision-level fusion techniques such as voting strategy, Bayesian method, behaviorknowledge space, and Dempster-Shafer theory [11]. Relatively speaking, there is maximum amount of information loss at decision-level.

This paper proposes a feature-level fusion method for rotating machinery fault diagnosis. Generally, heterogeneous information fusion is executed at feature-level fusion for mechanical condition monitoring and fault diagnosis in the present literature. For example, Barad et al. put forward the development of an ANN based model for condition monitoring of a power turbine that blends parameters belonging to performance, vibration, and lubrication [8]; Loutas et al. combined use of vibration, acoustic emission, and oil debris monitoring of rotating machinery [6]. The condition of mechanical system may be described in more detail by using heterogeneous information fusion, but this process needs multiclass sensors and its matching data acquisition systems, which would lead to higher monitoring costs and inconvenient operation of data acquisition in the real environment. ANN and SVM are the most popular classification models to execute decision at feature-level fusion $[12,13]$. The main difference between ANN and SVM is in their risk minimization. SVM is based on structural risk minimization principle, whereas ANN is based on traditional empirical risk minimization principle. The difference in risk minimization leads to a better generalization performance for SVM than that of ANN $[14,15]$. SVM is powerful for solving the problem with small sampling, nonlinear and high dimension in machinery condition classification. In this paper, the proposed feature-level fusion method belongs to homologous information fusion that the raw data all come from vibration sensors, so only a vibration testing system is needed for raw signal collected, which makes the process simpler. In this method, time-domain features are calculated from each vibration signal to compose a multidimensional feature set, and the SVM is selected as the classification model to process information fusion. In order to verify the effectiveness of the proposed method, fault diagnostic cases are tested, which include fault diagnosis of rolling bearing (identifying normal, inner race defect, outer race defect, and ball defect), fault diagnosis of gear (identifying normal, chipped tooth, and missing tooth), and fault diagnosis of rotor crack (identifying normal, crack depth of $3 \mathrm{~mm}$, and crack depth of $5 \mathrm{~mm}$ ). For each case study, the sensibilities of the features are analyzed.

\section{Theory}

2.1. Support Vector Machine (SVM). The SVM is a machine learning method based on the statistical learning theory and structural risk minimization principle. Given two category sample sets $\left(x_{i}, y_{i}\right)\left(x_{i} \in R^{d} ; y_{i} \in\{-1,+1\} ; i=1,2, \ldots, n\right), n$ is the number of samples. The optimal hyperplane separating the data can be obtained as a solution to the following optimization problem $[15,16]$ :

$$
\begin{array}{ll}
\operatorname{Min} & \frac{1}{2}\|\omega\|^{2}+C \sum_{i=1}^{n} \xi_{i} \\
\text { s.t. } & y_{i}\left[\left(\omega \cdot x_{i}\right)+b\right] \geq 1-\xi_{i} \quad\left(\xi_{i} \geq 0\right),
\end{array}
$$

where $\omega$ is weight vector, $b$ is scalar, $\xi_{i}$ is slack variable, and $C$ is error penalty.

The dual quadratic optimization description can be obtained by converting the problem with Kuhn-Tucker condition into the equivalent Lagrangian dual problem:

$$
\begin{array}{ll}
\operatorname{Max} & \sum_{i=0}^{n} \alpha_{i}-\frac{1}{2} \sum_{i=1}^{n} \sum_{j=1}^{n} \alpha_{i} \alpha_{j} y_{i} y_{j}\left(x_{i} \cdot x_{j}\right) \\
\text { s.t. } & \sum_{i=1}^{n} \alpha_{i} y_{i}=0 \quad\left(0 \leq \alpha_{i} \leq C\right),
\end{array}
$$

where $\alpha_{i}$ is Lagrange coefficient, which must meet the following equation:

$$
\alpha_{i}\left\{y_{i}\left[\left(\omega \cdot x_{i}\right)+b\right]-1+\xi_{i}\right\}=0
$$

The support vector is the sample which satisfies the equation $y_{i}\left[\left(\omega \cdot x_{i}\right)+b\right]=1-\xi_{i}$ at the time of the nonzero $\alpha_{i}$. It reveals that the samples at the edge of distribution are essential for classification. This leads to the optimal classification decision function:

$$
f(x)=\operatorname{sgn}\left\{\sum_{i=1}^{l} \alpha_{i}^{*} y_{i}\left(x_{i} \cdot x\right)+b^{*}\right\},
$$

where $l$ is the number of support vectors.

In linear inseparable condition, the samples $\left(x_{i}, y_{i}\right)\left(x_{i} \in\right.$ $\left.R^{d} ; y_{i} \in\{-1,+1\} ; i=1,2, \ldots, n\right)$ in input space are mapped into high dimensional space $H$ where the optimal classification surface can be established through the nonlinear mapping $\Phi: R^{d} \rightarrow H$. The nonlinear mapping $\Phi$ is usually difficult to be solved while kernel functions $K\left(x_{i}, x_{j}\right)$ 
meeting Mercer conditions can be used to solve this problem dexterously. The kernel function is described as follows:

$$
K\left(x_{i}, x_{j}\right)=\left\langle\Phi\left(x_{i}\right) \cdot \Phi\left(x_{j}\right)\right\rangle .
$$

The optimal classification decision function of linear inseparable samples is obtained using (5) into (4):

$$
\begin{aligned}
f(x) & =\operatorname{sgn}\left\{\sum_{i=1}^{l} \alpha_{i}^{*} y_{i}\left\langle\Phi\left(x_{i}\right) \cdot \Phi\left(x_{j}\right)\right\rangle+b^{*}\right\} \\
& =\operatorname{sgn}\left\{\sum_{i=1}^{l} \alpha_{i}^{*} y_{i} K\left(x_{i}, x_{j}\right)+b^{*}\right\} .
\end{aligned}
$$

The common kernel functions include linear kernel function, poly kernel function, radial basis function (RBF) kernel function, and sigmoid kernel function.

The traditional SVM was originally designed for binary classification problems. However, many practical problems in fault diagnosis field are multiclassification. Now some effective multiclass support vector machines were proposed which include "one-against-one," "one-against-all," directed acyclic graph (DAG), and so on [15]. Hsu et al. have given a comparison of these methods and pointed out that the "oneagainst-one" method is more suitable for practical use than other methods $[17,18]$.

2.2. Time-Domain Features. When the running conditions of the rotating machinery deviate from the normal condition, the time-domain statistical features of the vibration signal will be different from the normal condition. Furthermore, the time-domain statistical features will be also different under different defect models. Therefore, the time-domain statistics contain abundant defect information, and they can be used as sensitive character applied to fault diagnosis of rotating machinery. The time-domain statistical features used in this study are shown in Table 1.

2.3. Multisensors Information Fusion Model. The model of multisensor information fusion is used in this study and shown in Figure 1. The same character of different sensors is extracted to constitute a multidimensional vector and the SVM is used for pattern recognition. Twelve different timedomain features are analyzed one by one.

\section{Case Studies}

3.1. Data Acquisition. Experiments were performed on the machinery fault simulator (MFS) from SpectraQuest, Inc., shown in Figure 2. It can simulate most of faults that commonly occur in rotating machinery, such as rotor body defects, bearing defects, and gearbox defects. The shaft rotating speed was obtained by a laser speedometer. Acceleration signals were collected using the Dewetron 16 channels data acquisition system and IMI 608A11 accelerometers.

In the vibration testing experiments for roller bearing

\begin{tabular}{|c|c|c|}
\hline Code name & Feature & Equation \\
\hline$f_{1}$ & $\begin{array}{l}\text { Mean } \\
(\bar{x})\end{array}$ & $\bar{x}=\frac{1}{n} \sum\left|x_{i}\right|$ \\
\hline$f_{2}$ & $\begin{array}{l}\text { Peak } \\
\left(x_{p}\right)\end{array}$ & $x_{p}=\max \left(\left|x_{i}\right|\right)$ \\
\hline$f_{3}$ & $\begin{array}{l}\text { Amplitude square } \\
\left(x_{e}\right)\end{array}$ & $x_{e}=\sum_{i=1}^{n} x_{i}^{2}$ \\
\hline$f_{4}$ & $\begin{array}{l}\text { Root mean square } \\
\left(x_{\mathrm{rms}}\right)\end{array}$ & $x_{\mathrm{rms}}=\left(\frac{1}{n} \sum_{i=1}^{n} x_{i}^{2}\right)^{1 / 2}$ \\
\hline$f_{5}$ & $\begin{array}{l}\text { Root amplitude } \\
\left(x_{r}\right)\end{array}$ & $x_{r}=\left(\frac{1}{n} \sum_{i=1}^{n}\left|x_{i}\right|^{1 / 2}\right)^{2}$ \\
\hline$f_{6}$ & $\begin{array}{c}\text { Standard deviation } \\
\left(x_{\text {std }}\right)\end{array}$ & $x_{\text {std }}=\left(\frac{1}{n-1} \sum_{i=1}^{n}\left(x_{i}-\bar{x}\right)^{2}\right.$ \\
\hline$f_{7}$ & $\begin{array}{l}\text { Skewness } \\
\left(x_{\text {ske }}\right)\end{array}$ & $x_{\text {ske }}=\frac{\sum_{i=1}^{n}\left(x_{i}-\bar{x}\right)^{3}}{(n-1) x_{\text {std }}{ }^{3}}$ \\
\hline$f_{8}$ & $\begin{array}{c}\text { Kurtosis } \\
\left(x_{\text {kur }}\right)\end{array}$ & $x_{\mathrm{kur}}=\frac{\sum_{i=1}^{n}\left(x_{i}-\bar{x}\right)^{4}}{(n-1) x_{\mathrm{std}}^{4}}$ \\
\hline$f_{9}$ & $\begin{array}{l}\text { Waveform factor } \\
(\mathrm{SF})\end{array}$ & $\mathrm{SF}=\frac{x_{\mathrm{rms}}}{\bar{x}}$ \\
\hline$f_{10}$ & $\begin{array}{l}\text { Peak factor } \\
\quad(\mathrm{CF})\end{array}$ & $\mathrm{CF}=\frac{x_{p}}{x_{\mathrm{rms}}}$ \\
\hline$f_{11}$ & $\begin{array}{l}\text { Pulse factor } \\
\text { (IF) }\end{array}$ & $\mathrm{IF}=\frac{x_{p}}{\bar{x}}$ \\
\hline$f_{12}$ & $\begin{array}{l}\text { Margin factor } \\
\text { (CIF) }\end{array}$ & $\mathrm{CIF}=\frac{x_{p}}{x_{r}}$ \\
\hline
\end{tabular}
fault diagnosis, the simulator is composed of a motor,
TABLE 1: The statistic features in time domain.

$x_{i}$ in the table is discrete time series signal.

a coupling, a testing roller bearing fitted on the left of the shaft near the motor, a working roller bearing on the other side, a bearing load, and a shaft. The MFS provides a rolling bearing fault kit consisting of one normal, one inner race defect, one outer race defect, one with ball defect, and one combination of defects for performing experiments and studying bearing fault diagnosis. The acquisition frequency rate is $10 \mathrm{kHz}$. The sensors layout is depicted schematically in Figure 2(a) that a total of 8 sensors from $t_{1}$ to $t_{8}$ are used.

In the vibration testing experiments for gear fault diagnosis, the drive from the motor transmits to the gearbox through bearing-rotor system and belt. The gearbox consists of a two-stage parallel shaft with rolling bearings, helical gears, and a magnetic brake. The simplified diagram of gearbox transmission is shown in Figure 3 , where $z_{1}$ is the testing gear. The MFS provides a gear fault kit consisting of one normal, one chipped tooth, and one missing tooth for performing experiments and studying gear fault diagnosis. The acquisition frequency rate is $20 \mathrm{kHz}$. The sensors layout is depicted schematically in Figure 2(b) that a total of 8 sensors from $s_{1}$ to $s_{8}$ are used.

In the vibration testing experiments for rotor crack fault diagnosis, the rotor-bearing system is driven by the motor. In 


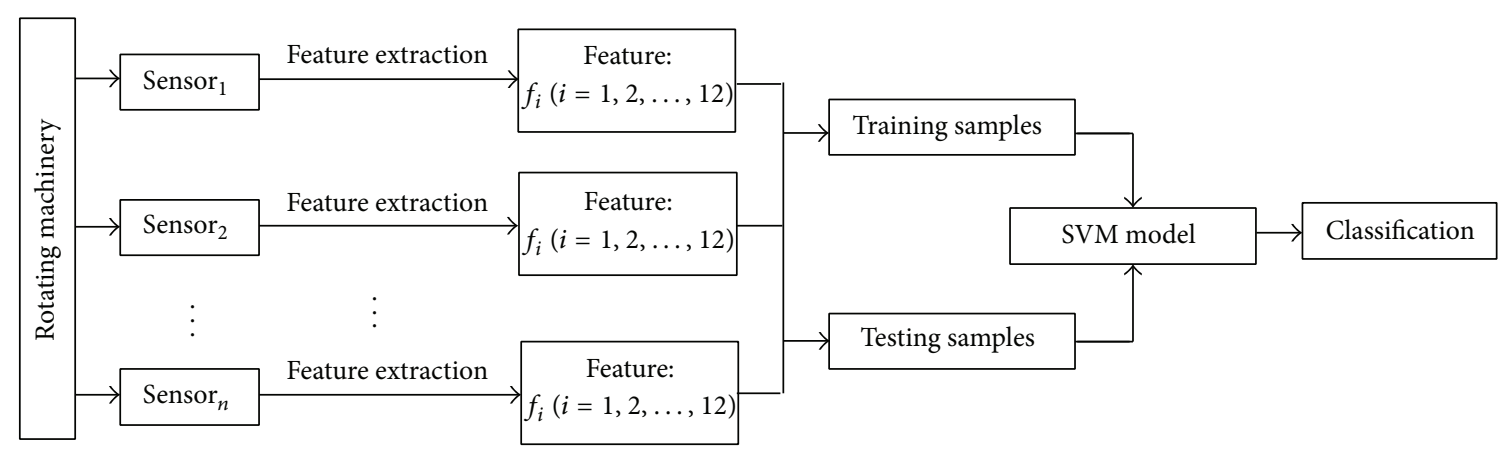

FIgURE 1: The multisensor information fusion process model.

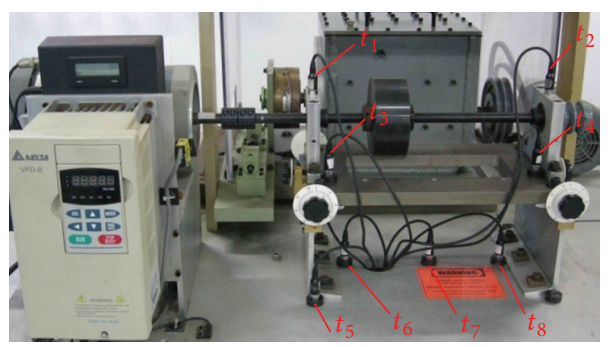

(a) The front view

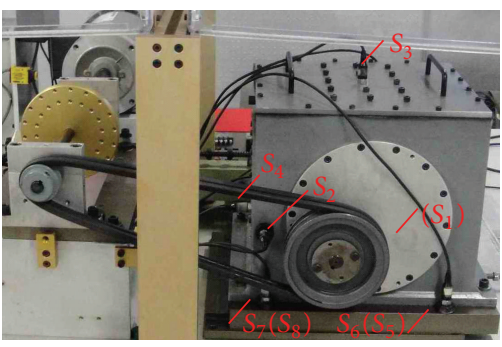

(b) The side view

FIGURE 2: The machinery fault simulator.

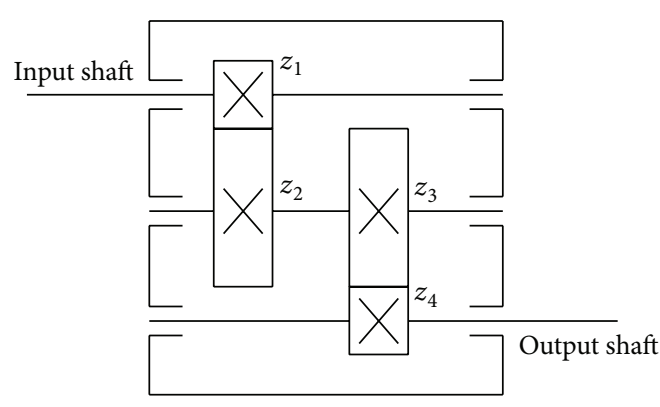

FIgURE 3: The simplified diagram of gearbox transmission.

order to simulate the expanding of crack, crack faults were introduced to the test rotor by using the electrodischarge machining. The defect with crack width of $0.12 \mathrm{~mm}$ and crack depth of $3 \mathrm{~mm}$ represents slight defect, and that with crack width of $0.12 \mathrm{~mm}$ and crack depth of $5 \mathrm{~mm}$ represents serious defect. The acquisition frequency rate is $10 \mathrm{kHz}$. The sensors layout is depicted schematically in Figure 2(a) that a total of 4 sensors from $t_{1}$ to $t_{4}$ are used.

3.2. Fault Diagnostic Case of Gear. Vibration signals of gear with three fault models including normal, chipped tooth, and missing tooth are taken for analysis. A certain timedomain feature is calculated from eight sensors $\left(s_{1}\right.$ to $\left.s_{8}\right)$ to constitute an eight-dimensional vector as a fault sample. One hundred and ten fault samples from each model, a total of three hundred and thirty samples, are used to constitute the fault sample sets. Sixty fault samples from each model, a total of one hundred and eighty samples, are selected randomly as training samples and the others are used as testing samples. Twelve time-domain statistics are analyzed one by one.

LibSVM-mat-2.9 is chosen for SVM calculation. LibSVM is developed by Lin Chih-Jen from Taiwan [19]. It is a simple and easy-to-use SVMs tool for classification. RBF kernel function is chosen as kernel function shown as follows:

$$
K\left(x_{i}, x_{j}\right)=\exp \left(-g\left\|x_{i}-x_{j}\right\|^{2}\right), \quad g>0 .
$$

The cross-validation combination with network search method is used to search the best parameters: the error penalty $C$ of SVM and $g$ of RFB. One-against-one multiclassification is chosen for pattern recognition. The diagnostic results of gear by using different time-domain features are listed in Table 2.

It can be found from Table 2 that the highest diagnostic accuracy is $93.33 \%$ by using the peak factor as feature to constitute fusional vector for gear fault diagnosis. Sensitivity of the features can be indicated by diagnostic accuracy when using the same classifier SVM, so, the peak factor is the most sensitive feature in the twelve time-domain features for identifying gear defect, followed by the amplitude square, root amplitude, mean, root mean square, standard deviation, and peak. The diagnostic accuracy is all above $80 \%$ by using these features. The skewness, kurtosis, waveform factor, and margin factor are less sensitive comparatively. The diagnostic accuracy is all under $70 \%$ by using these features. 
TABLE 2: Diagnostic results of gear by using different features for fusion.

\begin{tabular}{|c|c|c|c|c|c|c|}
\hline \multirow{2}{*}{ Feature } & \multicolumn{2}{|c|}{ The best parameter } & \multicolumn{4}{|c|}{ Diagnostic accuracy (\%) } \\
\hline & C & $g$ & Normal & Chipped tooth & Missing tooth & All testing samples \\
\hline Mean & $2^{6.5}$ & $2^{15}$ & 98 & 82 & 84 & 88.00 \\
\hline Peak & $2^{1}$ & $2^{8}$ & 98 & 86 & 66 & 83.33 \\
\hline Amplitude square & $2^{12}$ & $2^{0}$ & 98 & 82 & 88 & 89.33 \\
\hline Root mean square & $2^{14}$ & $2^{3}$ & 98 & 78 & 86 & 87.33 \\
\hline Root amplitude & $2^{11.5}$ & $2^{11.5}$ & 100 & 88 & 76 & 88.00 \\
\hline Standard deviation & $2^{7.5}$ & $2^{9.5}$ & 98 & 78 & 86 & 87.33 \\
\hline Skewness & $2^{8}$ & $2^{-1}$ & 98 & 30 & 58 & 62.00 \\
\hline Kurtosis & $2^{2.5}$ & $2^{-3}$ & 94 & 68 & 40 & 67.33 \\
\hline Waveform factor & $2^{-1.5}$ & $2^{9}$ & 92 & 42 & 30 & 55.33 \\
\hline Peak factor & $2^{0.5}$ & $2^{-1}$ & 94 & 92 & 94 & 93.33 \\
\hline Pulse factor & $2^{-0.5}$ & $2^{-2}$ & 96 & 64 & 60 & 73.33 \\
\hline Margin factor & $2^{0}$ & $2^{-3}$ & 96 & 57 & 55 & 69.33 \\
\hline
\end{tabular}

TABLE 3: Diagnostic results of gear by using different single sensors.

\begin{tabular}{|c|c|c|c|c|c|c|}
\hline \multirow{2}{*}{ Sensor } & \multicolumn{2}{|c|}{ The best parameter } & \multicolumn{4}{|c|}{ Diagnostic accuracy (\%) } \\
\hline & $C$ & $g$ & Normal & Chipped tooth & Missing tooth & All testing samples \\
\hline$s_{1}$ & $2^{5.5}$ & $2^{8}$ & 40 & 28 & 86 & 51.33 \\
\hline$s_{2}$ & $2^{3.5}$ & $2^{13.5}$ & 22 & 94 & 100 & 72.00 \\
\hline$s_{3}$ & $2^{1}$ & $2^{4}$ & 84 & 82 & 76 & 80.67 \\
\hline$s_{4}$ & $2^{3}$ & $2^{8.5}$ & 54 & 80 & 94 & 76.00 \\
\hline$s_{5}$ & $2^{15}$ & $2^{0.5}$ & 64 & 90 & 100 & 84.67 \\
\hline$s_{6}$ & $2^{13.5}$ & $2^{6.5}$ & 92 & 96 & 56 & 81.33 \\
\hline$s_{7}$ & $2^{11.5}$ & $2^{7.5}$ & 90 & 96 & 64 & 83.33 \\
\hline$s_{8}$ & $2^{15}$ & $2^{4}$ & 78 & 86 & 58 & 74.00 \\
\hline
\end{tabular}

It also can be found from Table 2 that the accuracy of normal testing samples is all above $90 \%$ by using any feature. During the analysis, we also found that the samples of defect with chipped tooth and defect with missing tooth are easy to be misclassified with each other, but defect samples are seldom mistakenly regarded as normal samples, so it can be deduced that normal and defect gear are always easy to distinguish.

In order to compare with single sensor for gear fault diagnosis, take eight features from a single sensor to constitute an eight-dimensional vector as a fault sample. The eight features are the peak factor, amplitude square, root amplitude, mean, root mean square, standard deviation, peak, and pulse factor, which are the first eight sensitive features for identifying gear defect selected on the basis of the above analysis result. In order to avoid the orders of magnitude difference of different features, normalized eigenvector is processed before inputting SVM. In fact, during the proposed multisensors information analysis, the fault sample is constituted by the same feature from multisensors, so the orders of magnitude difference are nonexistent and normalized eigenvector is not needed. The sensors $s_{1}$ to $s_{8}$ are analyzed one by one.
The diagnostic results of gear by using different single sensors are listed in Table 3.

Comparing with Tables 2 and 3, it can be found that there is higher diagnostic accuracy by using multisensors information fusion method than using single sensor method as a whole.

3.3. Fault Diagnostic Case of Rolling Bearing. Vibration signals of rolling bearing with four fault models including normal, inner race defect, outer race defect, and ball defect are taken for analysis. A certain time-domain feature is calculated from eight sensors $\left(t_{1}\right.$ to $\left.t_{8}\right)$ to constitute an eightdimensional vector as a fault sample. One hundred and ten fault samples from each model, a total of four hundred and forty samples, are used to constitute the fault sample sets. Fifty fault samples from each model, a total of two hundred samples, are selected randomly as training samples and the others are used as testing samples. Twelve timedomain statistics are analyzed one by one.

LibSVM-mat-2.9 is chosen for SVM calculation. Gaussian kernel function is chosen as kernel function. The crossvalidation combination with network search method is used 
TABLE 4: Diagnostic results of rolling bearing by using different features for fusion.

\begin{tabular}{|c|c|c|c|c|c|c|c|}
\hline \multirow{2}{*}{ Feature } & \multicolumn{2}{|c|}{ The best parameter } & \multicolumn{5}{|c|}{ Diagnostic accuracy (\%) } \\
\hline & $C$ & $g$ & Normal & Inner race defect & Outer race defect & Ball defect & All testing samples \\
\hline Mean & $2^{-3}$ & $2^{15}$ & 100 & 100 & 100 & 100 & 100 \\
\hline Peak & $2^{3}$ & $2^{7}$ & 94.29 & 94.29 & 100 & 100 & 97.14 \\
\hline Amplitude square & $2^{-3}$ & $2^{5}$ & 100 & 100 & 100 & 100 & 100 \\
\hline Root mean square & $2^{4.5}$ & $2^{15}$ & 100 & 100 & 100 & 100 & 100 \\
\hline Root amplitude & $2^{-2}$ & $2^{15}$ & 100 & 100 & 100 & 100 & 100 \\
\hline Standard deviation & $2^{4.5}$ & $2^{15}$ & 100 & 100 & 100 & 100 & 100 \\
\hline Skewness & $2^{2}$ & $2^{4}$ & 65.71 & 84.29 & 62.86 & 80 & 73.21 \\
\hline Kurtosis & $2^{6}$ & $2^{-2}$ & 90.00 & 72.86 & 82.86 & 97.14 & 85.71 \\
\hline Waveform factor & $2^{2}$ & $2^{9}$ & 81.43 & 70.00 & 80.00 & 98.57 & 82.50 \\
\hline Peak factor & $2^{3}$ & $2^{-3}$ & 64.29 & 64.29 & 78.57 & 95.71 & 75.71 \\
\hline Pulse factor & $2^{2}$ & $2^{-3}$ & 71.43 & 64.29 & 81.43 & 95.71 & 78.21 \\
\hline Margin factor & $2^{5}$ & $2^{-4.5}$ & 71.43 & 68.57 & 80 & 95.71 & 78.93 \\
\hline
\end{tabular}

TABLE 5: Diagnostic results of rolling bearing by using different single sensors.

\begin{tabular}{|c|c|c|c|c|c|c|c|}
\hline \multirow{2}{*}{ Sensor } & \multicolumn{2}{|c|}{ The best parameter } & \multicolumn{5}{|c|}{ Diagnostic accuracy (\%) } \\
\hline & $C$ & $g$ & Normal & Inner race defect & Outer race defect & Ball defect & All testing samples \\
\hline$t_{1}$ & $2^{14.5}$ & $2^{-1.5}$ & 85.56 & 88.89 & 100 & 98.89 & 93.33 \\
\hline$t_{2}$ & $2^{11}$ & $2^{5.5}$ & 58.89 & 58.89 & 100 & 100 & 79.44 \\
\hline$t_{3}$ & $2^{3.5}$ & $2^{4}$ & 96.67 & 78.89 & 100 & 98.89 & 93.67 \\
\hline$t_{4}$ & $2^{14.5}$ & $2^{0.5}$ & 100 & 85.56 & 83.33 & 98.89 & 91.94 \\
\hline$t_{5}$ & $2^{2}$ & $2^{7.5}$ & 100 & 96.67 & 100 & 100 & 99.17 \\
\hline$t_{6}$ & $2^{2}$ & $2^{7.5}$ & 97.7 & 96.67 & 100 & 91.11 & 96.39 \\
\hline$t_{7}$ & $2^{11}$ & $2^{3.5}$ & 98.89 & 90 & 100 & 88.89 & 94.44 \\
\hline$t_{8}$ & $2^{10}$ & $2^{-1.5}$ & 61.11 & 51.11 & 53.33 & 87.78 & 63.33 \\
\hline
\end{tabular}

to search the parameters $C$ and $g$. One-against-one multiclassification is chosen for pattern recognition. The diagnostic results of rolling bearing by using different time-domain features are listed in Table 4.

It can be found from Table 4 that the mean, amplitude square, root mean square, root amplitude, and standard deviation are the first five sensitive features for identifying rolling bearing defect. The diagnostic accuracy is all $100 \%$ by using these features. Comparing with Tables 4 and 2, it can be found that there is a higher diagnostic accuracy for rolling bearing fault diagnosis than for gear fault diagnosis by using the proposed information fusion method as a whole. The main cause is that the way from the defect position of rolling bearing to the sensor installation position is shorter and simpler than the way from the defect position of gear.

In order to compare with single sensor for rolling bearing fault diagnosis, take eight features from a single sensor to constitute an eight-dimensional vector as a fault sample. The eight features are the mean, amplitude square, root mean square, root amplitude, standard deviation, peak, kurtosis, and waveform factor, which are the first eight sensitive features for identifying rolling bearing defect selected on the basis of the above analysis result. In order to avoid the orders of magnitude difference of different features, normalized eigenvector is processed before inputting SVM. The sensors $t_{1}$ to $t_{8}$ are analyzed one by one. The diagnostic results of rolling bearing by using different single sensor are listed in Table 5 .

Comparing with Tables 4 and 5, it can be found that there is higher diagnostic accuracy by using multisensors information fusion method than using single sensor method as a whole.

3.4. Fault Diagnostic Case of Rotor Crack. Vibration signals of rotor crack with three fault models including normal, crack depth of $3 \mathrm{~mm}$, and crack depth of $5 \mathrm{~mm}$ are taken for analysis. A certain time-domain feature is calculated from four sensors $\left(t_{1}\right.$ to $\left.t_{4}\right)$ to constitute a four-dimensional vector as a fault sample. One hundred fault samples from each model, a total of three hundred samples, are used to constitute the fault sample sets. Fifty fault samples from each model, total of one hundred and fifty samples, are selected randomly as training samples and the others are used as testing samples. Twelve time-domain statistics are analyzed one by one.

LibSVM-mat-2.9 is chosen for SVM calculation. Gaussian kernel function is chosen as kernel function. The crossvalidation combination with network search method is used to search the parameters $C$ and $g$. One-against-one multiclassification is chosen for pattern recognition. The diagnostic 
TABLE 6: Diagnostic results of rotor crack by using different features for fusion.

\begin{tabular}{|c|c|c|c|c|c|c|}
\hline \multirow{2}{*}{ Feature } & \multicolumn{2}{|c|}{ The best parameter } & \multicolumn{4}{|c|}{ Diagnostic accuracy (\%) } \\
\hline & $C$ & $g$ & Normal & Crack depth of $3 \mathrm{~mm}$ & Crack depth of $5 \mathrm{~mm}$ & All testing samples \\
\hline Mean & $2^{4.5}$ & $2^{12}$ & 98 & 94 & 100 & 98.67 \\
\hline Peak & $2^{0}$ & $2^{9.5}$ & 72 & 86 & 88 & 85.33 \\
\hline Amplitude square & $2^{7}$ & $2^{2}$ & 100 & 96 & 94 & 96.67 \\
\hline Root mean square & $2^{6}$ & $2^{4}$ & 100 & 98 & 100 & 99.67 \\
\hline Root amplitude & $2^{5.5}$ & $2^{3}$ & 100 & 92 & 100 & 97.33 \\
\hline Standard deviation & $2^{4}$ & $2^{8}$ & 98 & 96 & 98 & 98.67 \\
\hline Skewness & $2^{5}$ & $2^{-2}$ & 58 & 44 & 46 & 58.00 \\
\hline Kurtosis & $2^{3.5}$ & $2^{-1}$ & 44 & 74 & 84 & 71.00 \\
\hline Waveform factor & $2^{2}$ & $2^{3}$ & 34 & 80 & 84 & 72.33 \\
\hline Peak factor & $2^{2.5}$ & $2^{-1.5}$ & 42 & 46 & 62 & 69.33 \\
\hline Pulse factor & $2^{-3}$ & $2^{-1}$ & 28 & 74 & 76 & 64.00 \\
\hline Margin factor & $2^{1}$ & $2^{-3}$ & 32 & 72 & 74 & 64.67 \\
\hline
\end{tabular}

results of gear by using different time-domain features are listed in Table 6.

It can be found from Table 5 that the mean, amplitude square, root mean square, root amplitude, and standard deviation are the first five sensitive features for identifying rotor crack defect. The diagnostic accuracy is all $90 \%$ by using these features. The result is similar to fault diagnostic case of rolling bearing.

\section{Conclusion}

In this paper, a feature-level information fusion methodology is proposed that all the features are calculated using vibration data in time domain to constitute fusional vector and the SVM is used for classification. Only a vibration testing system is needed for raw signal collected in this method, so the process is simpler. The effectiveness of the proposed methodology is tested with examples of gear, rolling bearing, and rotor crack fault diagnosis. Sensitivities of the twelve time-domain features are discussed in each case study. The analyzed results indicate that the peak factor is the most sensitive feature in the twelve time-domain features for identifying gear defect, but it is not very sensitive for identifying rolling bearing and rotor crack defect. The mean, amplitude square, root mean square, root amplitude, and standard deviation are all sensitive for identifying gear, rolling bearing, and rotor crack defect comparatively.

The features used and discussed in this paper are all in time domain; however, features in frequency domain also can be used for fault diagnosis of rotating machinery and the sensibilities of the features for identifying rolling bearing, gear, and rotor defect are also worth studying in the future.

\section{Conflict of Interests}

The authors declare that there is no conflict of interests regarding the publication of this paper.

\section{Acknowledgments}

This work is supported by the National Natural Science Foundation of China (51105138 and 51175169), the National High Technology Research and Development Program Items (2012AA041805), the Pre-research Project (813040302), the CEEUSRO special plan of Hunan province (2010XK6066), and the Aid Program for Science and Technology Innovative Research Team in Higher Educational Institutions of Hunan province.

\section{References}

[1] V. T. Tran and B.-S. Yang, "An intelligent condition-based maintenance platform for rotating machinery," Expert Systems with Applications, vol. 39, no. 3, pp. 2977-2988, 2012.

[2] K. P. Kumar, K. V. N. S. Rao, K. R. Krishna, and B. Theja, "Neural network based vibration analysis with novelty in data detection for a large steam turbine," Shock and Vibration, vol. 19, no. 1, pp. 25-35, 2012.

[3] L. L. Jiang, Y. L. Liu, X. J. Li, and S. Tang, "Using bispectral distribution as a feature for rotating machinery fault diagnosis," Measurement, vol. 44, no. 7, pp. 1284-1292, 2011.

[4] C. Z. Han, H. Y. Zhu, and Z. S. Duan, Multi-Source Information Fusion, Tsinghua University Press, Beijing, China, 2006.

[5] G. F. Bin, J. J. Gao, X. J. Li, and B. S. Dhillon, "Early fault diagnosis of rotating machinery based on wavelet packetsempirical mode decomposition feature extraction and neural network," Mechanical Systems and Signal Processing, vol. 27, no. 1, pp. 696-711, 2012.

[6] T. H. Loutas, D. Roulias, E. Pauly, and V. Kostopoulos, "The combined use of vibration, acoustic emission and oil debris online monitoring towards a more effective condition monitoring of rotating machinery," Mechanical Systems and Signal Processing, vol. 25, no. 4, pp. 1339-1352, 2011.

[7] G. Niu, T. Han, B.-S. Yang, and A. C. C. Tan, "Multi-agent decision fusion for motor fault diagnosis," Mechanical Systems and Signal Processing, vol. 21, no. 3, pp. 1285-1299, 2007.

[8] S. G. Barad, P. V. Ramaiah, R. K. Giridhar, and G. Krishnaiah, "Neural network approach for a combined performance and 
mechanical health monitoring of a gas turbine engine," Mechanical Systems and Signal Processing, vol. 27, no. 1, pp. 729-742, 2012.

[9] Q. Tan and Y.-H. Xiang, "Application of weighted evidential theory and its information fusion method in fault diagnosis," Journal of Vibration and Shock, vol. 27, no. 4, pp. 112-116, 2008.

[10] Y.-Y. Liu, Y.-F. Ju, C.-D. Duan, and X.-F. Zhao, "Structure damage diagnosis using neural network and feature fusion," Engineering Applications of Artificial Intelligence, vol. 24, no. 1, pp. 87-92, 2011.

[11] O. Basir and X. Yuan, "Engine fault diagnosis based on multisensor information fusion using Dempster-Shafer evidence theory," Information Fusion, vol. 8, no. 4, pp. 379-386, 2007.

[12] G. Niu and B.-S. Yang, "Intelligent condition monitoring and prognostics system based on data-fusion strategy," Expert Systems with Applications, vol. 37, no. 12, pp. 8831-8840, 2010.

[13] A. Ghasemloonia and S. Esmaeel Zadeh Khadem, "Gear tooth failure detection by the resonance demodulation technique and the instantaneous power spectrum method-a comparative study," Shock and Vibration, vol. 18, no. 3, pp. 503-523, 2011.

[14] Z. S. Chen and Y. M. Yang, "Fault diagnostics of helicopter gearboxes based on multi-sensor mixtured hidden Markov models," Journal of Vibration and Acoustics, Transactions of the ASME, vol. 134, no. 3, Article ID 031010, 2012.

[15] A. Widodo and B.-S. Yang, "Support vector machine in machine condition monitoring and fault diagnosis," Mechanical Systems and Signal Processing, vol. 21, no. 6, pp. 2560-2574, 2007.

[16] B.-S. Yang, T. Han, and W.-W. Hwang, "Fault diagnosis of rotating machinery based on multi-class support vector machines," Journal of Mechanical Science and Technology, vol. 19, no. 3, pp. 846-859, 2005.

[17] J. Y. Yang, Y. Y. Zhu, Y. S. Zhang, and Q. Wang, "Intelligent fault diagnosis of rolling element bearing based on SVMS and statistical characteristics," in Proceedings of the ASME International Conference on Manufacturing Science and Engineering, pp. 525536, Atlanta, Ga, USA, October 2007.

[18] C.-W. Hsu and C.-J. Lin, "A comparison of methods for multiclass support vector machines," IEEE Transactions on Neural Networks, vol. 13, no. 2, pp. 415-425, 2002.

[19] C. Hsu, C. C. Chang, and C. J. Lin, "A practical guide to support vector classification[EB/OL]," 2009, http://www.csie .ntu.edu.tw/ cjlin/. 

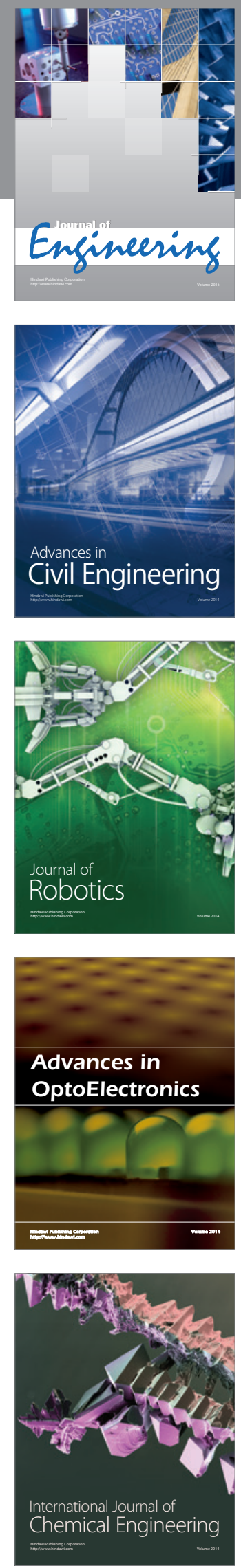

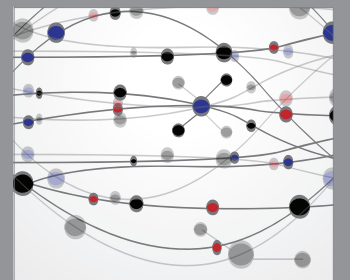

The Scientific World Journal
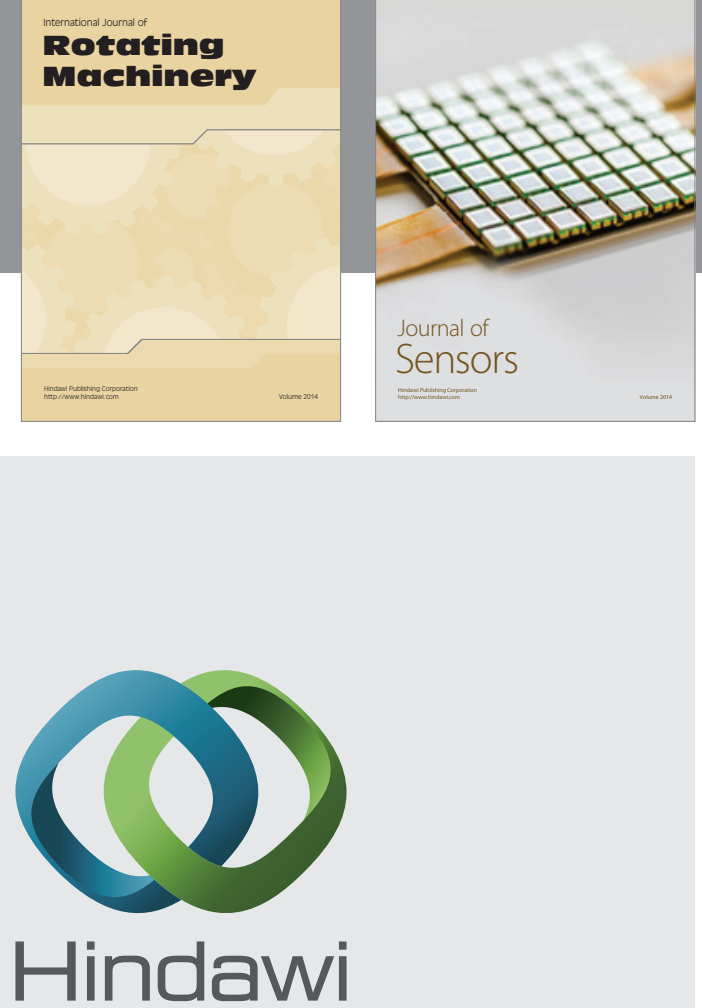

Submit your manuscripts at http://www.hindawi.com
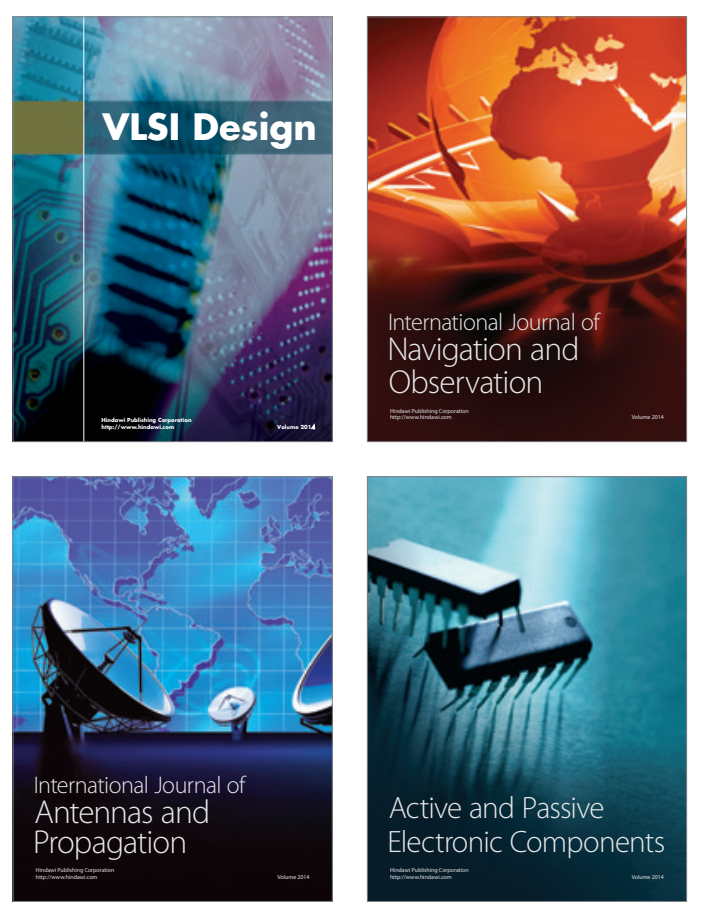
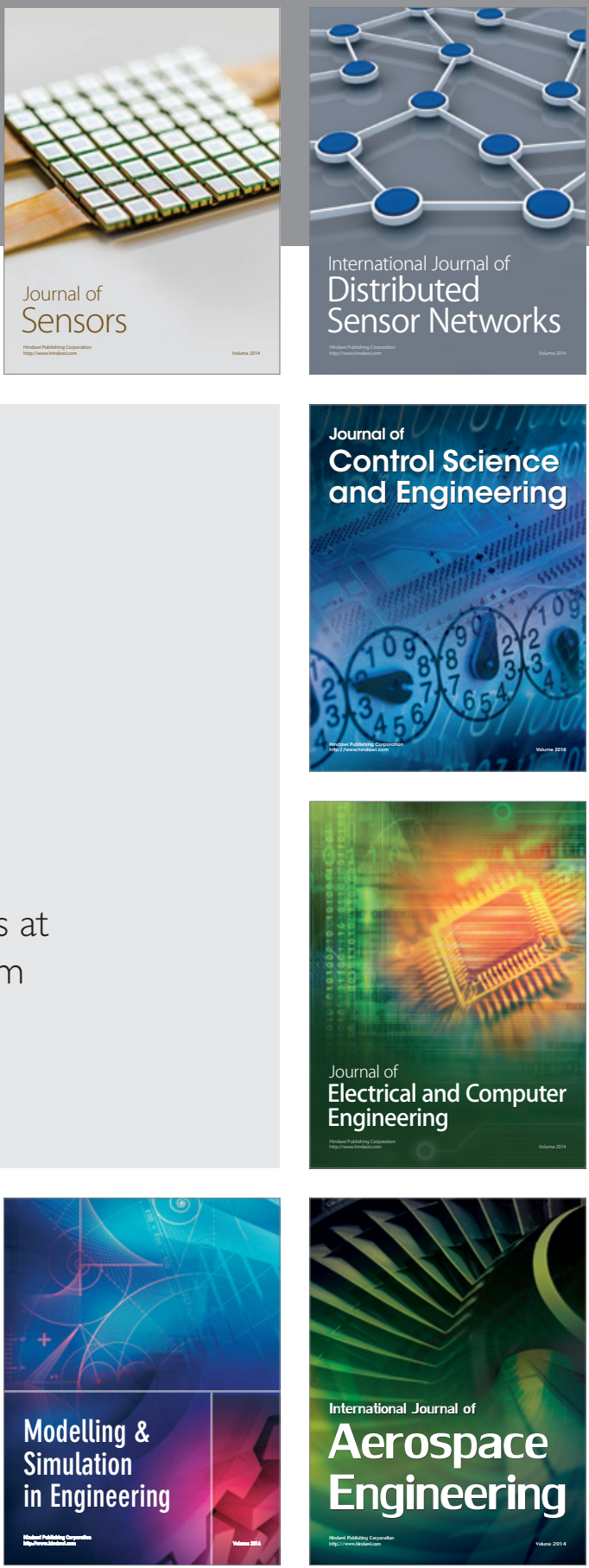

Journal of

Control Science

and Engineering
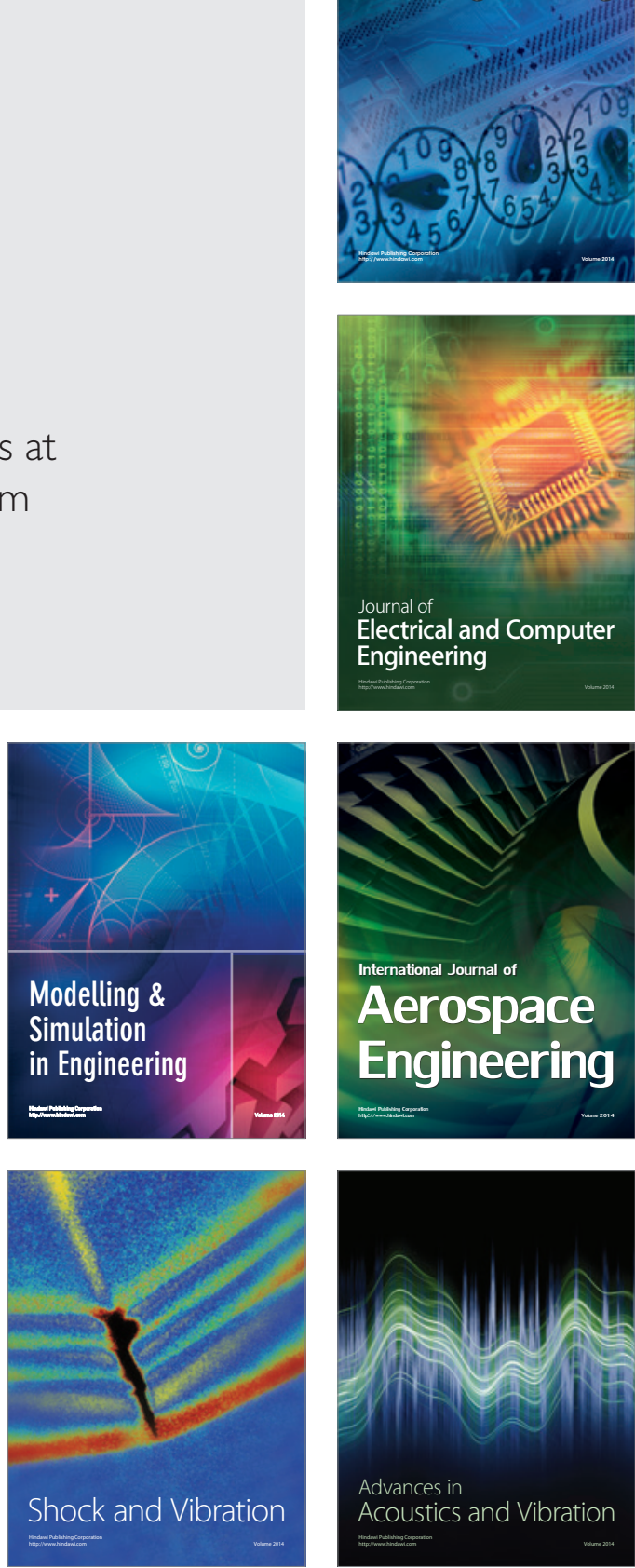